\title{
Strings on NS-NS backgrounds as integrable deformations
}

\author{
Marco Baggio ${ }^{1, *}$ and Alessandro Sfondrini ${ }^{2, \dagger}$ \\ ${ }^{1}$ Instituut voor Theoretische Fysica, KU Leuven, Celestijnenlaan 200D, B-3001 Leuven, Belgium \\ ${ }^{2}$ Institut für theoretische Physik, ETH Zürich, Wolfgang-Pauli-Straße 27, 8093 Zürich, Switzerland
}

(Received 11 April 2018; published 19 July 2018)

\begin{abstract}
We consider the world-sheet $S$ matrix of superstrings on an $\mathrm{AdS}_{3} \times \mathrm{S}^{3} \times \mathrm{T}^{4} \mathrm{NS}-\mathrm{NS}$ background in uniform light-cone gauge. We argue that scattering is given by a CDD factor that is nontrivial only between opposite-chirality particles, yielding a spin-chain-like Bethe ansatz. Our proposal reproduces the spectrum of nonprotected states computed from the Wess-Zumino-Witten description and the perturbative tree-level $\mathrm{S}$ matrix. This suggests that the model is an integrable deformation of a free theory similar to those arising from the $T \bar{T}$ composite operator.
\end{abstract}

DOI: 10.1103/PhysRevD.98.021902

\section{INTRODUCTION}

The study of the AdS/CFT correspondence [1-3] gave new energy to the search for exactly solvable string backgrounds. For a general string background only, few observables may be computed exactly, usually owing to supersymmetry. Notable exceptions are plane-wave backgrounds [4-6] where the light-cone Hamiltonian is free, Wess-Zumino-Witten (WZW) models [7-10] where current algebras can be used to solve the theory, and integrable backgrounds [11-13] where the world-sheet scattering in light-cone gauge factorizes along the lines of Ref. [14]. The best-understood integrable AdS background is $\mathrm{AdS}_{5} \times \mathrm{S}^{5}$ supported by Ramond-Ramond (R-R) five-form fluxes. The string equations of motion are integrable [15] and the factorized $\mathrm{S}$ matrix can be computed from symmetry considerations [16-18]. The spectrum follows from imposing periodic boundary conditions and accounting for finite-size "wrapping" effects [19], leading eventually to a quantum spectral curve [20]. Remarkably, the integrability approach to $\mathrm{AdS}_{5} \times \mathrm{S}^{5}$ superstrings can be extended to three- [21] and higher-point [22,23] correlation functions, and even to nonplanar corrections [24,25], though the treatment of wrapping corrections is less thoroughly understood in that context. Another important class of integrable backgrounds is given by $\mathrm{AdS}_{3} \times \mathrm{S}^{3} \times \mathrm{T}^{4}$ and $\mathrm{AdS}_{3} \times \mathrm{S}^{3} \times$ $S^{3} \times S^{1}$ geometries supported by R-R and Neveu-SchwarzNeveu-Schwarz (NS-NS) three-form fluxes. They are also classically integrable $[26,27]$ and their $\mathrm{S}$ matrix can be

\footnotetext{
*marco.baggio@kuleuven.be

sfondria@itp.phys.ethz.ch
}

Published by the American Physical Society under the terms of the Creative Commons Attribution 4.0 International license. Further distribution of this work must maintain attribution to the author(s) and the published article's title, journal citation, and DOI. Funded by SCOAP. fixed by symmetries [28-30], even for mixed background fluxes [31,32]. The purely R-R backgrounds resemble $\mathrm{AdS}_{5} \times \mathrm{S}^{5}$ : the $\mathrm{S}$ matrix has a complicated scalar factor $[33,34]$ and the dispersion relation is periodic [28] suggesting a dual spin-chain interpretation similar to Ref. [35]. Instead the NS-NS flux yields a linear contribution to the dispersion $[31,36]$. The pure-NS-NS model corresponds to a supersymmetric WZW model and the S matrix should simplify drastically there. The analysis of light-cone gauge symmetries of Ref. [31], valid for generic mixtures of R-R and NS-NS fluxes, is insufficient to determine the $\mathrm{S}$ matrix at the WZW point. In this article, we analyze the $\mathrm{AdS}_{3} \times$ $\mathrm{S}^{3} \times \mathrm{T}^{4}$ WZW model in uniform light-cone gauge [37-39], considering its classical bosonic Hamiltonian, its spectrum and its $\mathrm{S}$ matrix. We observe that shifting the gauge parameter has a similar effect to a $T \bar{T}$ deformation [40-42] and that the $\mathrm{T}^{4}$ sector of the theory is free in a suitable gauge. This motivates us to further investigate the spectrum to determine if it can be related to that of a free theory. We find that in the "spectrally unflowed" sector [10] the energies of nonprotected states can be reproduced from the Bethe-Yang equations by adding a CDD factor [43] to a free theory. The resulting $S$ matrix coincides at tree level with the known perturbative result [44] in a suitable gauge. Moreover, we argue that, due to supersymmetry, wrapping corrections cancel out similarly to what happened for protected states in Ref. [45], so that the Bethe-Yang equations are exact. The CDD factor appearing in our construction is exactly that of a $T \bar{T}$ deformation when we restrict to $\mathrm{T}^{4}$ [46]; in general however it differs from it due to the presence of an additional $\mathrm{u}(1)$ current. The simple form of the S matrix makes the study of this NS-NS background almost as straightforward as that of a planewave one, paving the way to a wealth of explicit computations. We conclude this article by detailing additional checks of our proposal, which we intend to present in an 
upcoming publication [47], as well as by commenting on several possible future directions.

\section{WZW MODEL IN LIGHT-CONE GAUGE}

The Green-Schwarz action for $\mathrm{AdS}_{3} \times \mathrm{S}^{3} \times \mathrm{T}^{4}$ with mixed NS-NS and R-R three-form fluxes has been analyzed in some detail in Ref. [31]. We restrict to the pure NS-NS case corresponding to the WZW model, and find the bosonic action

$$
\mathbf{S}=-\frac{k}{4 \pi} \int_{-\infty}^{+\infty} \mathrm{d} \tau \int_{0}^{R} \mathrm{~d} \sigma\left(\gamma^{\alpha \beta} G_{\mu \nu}+\epsilon^{\alpha \beta} B_{\mu \nu}\right) \partial_{\alpha} X^{\mu} \partial_{\beta} X^{\nu},
$$

where $k$ is the WZW level, $\gamma^{\alpha \beta}$ is the world-sheet metric with $|\gamma|=-1, G_{\mu \nu}$ is the $\mathrm{AdS}_{3} \times \mathrm{S}^{3} \times \mathrm{T}^{4}$ metric and $B_{\mu \nu}$ is the Kalb-Ramond field. We write the line element as

$$
\begin{aligned}
\mathrm{d} s^{2}= & -\left(1+|z|^{2}\right) \mathrm{d} t^{2}+\left(1-|y|^{2}\right) \mathrm{d} \phi^{2}+\mathrm{d} x^{j} \mathrm{~d} x^{j} \\
& +\left(\delta_{i j}-\frac{z_{i} z_{j}}{1+|z|^{2}}\right) \mathrm{d} z^{i} \mathrm{~d} z^{j}+\left(\delta_{i j}+\frac{y_{i} y_{j}}{1-|y|^{2}}\right) \mathrm{d} y^{i} \mathrm{~d} y^{j},
\end{aligned}
$$

where $t, z_{1}, z_{2}$ are in $\mathrm{AdS}_{3}, \phi, y^{3}, y^{4}$ describe $S^{3}$ and $x^{5}, \ldots x^{8}$ give $\mathrm{T}^{4}$. The Kalb-Ramond field is given by $B=\epsilon^{i j} z_{i} d z_{j} \wedge d t-\epsilon^{i j} y_{i} d y_{j} \wedge d \phi$. Fermions couple to $H=d B$, see Refs. [31,48,49] for explicit formulas. To fix uniform light-cone gauge [37-39], we introduce, for $0 \leq a \leq 1$,

$$
x^{+}=(1-a) t+a \phi, \quad x^{-}=\phi-t,
$$

and following, e.g., Ref. [11], we introduce conjugate momenta $p_{\mu}=\delta \mathbf{S} / \delta\left(\partial_{0} X^{\mu}\right)$ and fix

$$
x^{+}=\tau, \quad p_{-}=(1-a) p_{\phi}-a p_{t}=1 .
$$

This breaks conformal invariance and, in particular, fixes the world-sheet size $R$

$$
R=(1-a) \int_{0}^{R} \mathrm{~d} \sigma p_{\phi}-a \int_{0}^{R} \mathrm{~d} \sigma p_{t}=J+a(E-J),
$$

where $E$ is the string energy and $J$ its angular momentum. The light-cone Hamiltonian is

$$
\mathbf{H}=-\int_{0}^{R} \mathrm{~d} \sigma p_{+}=E-J,
$$

and the $\mathrm{AdS}_{3} \times \mathrm{S}^{3}$ BPS bound guarantees $\mathbf{H} \geq 0$. Notice that $-p_{+}$is $a$-dependent, and gauge invariance dictates

$$
\frac{\mathrm{d}}{\mathrm{d} a} \int_{0}^{R(a)} \mathrm{d} \sigma p_{+}(a)=0 .
$$

The density $p_{+}(a)$ can be easily found as in Ref. [31] by solving the Virasoro constraints. Truncating it to $\mathrm{T}^{4}$ modes and setting $s=(a-1 / 2)$, we find

$$
\left.\mathbf{H}\right|_{\mathrm{T}^{4}}=\int_{0}^{R(s)} \mathrm{d} \sigma \frac{1-\sqrt{1-4 s H_{\text {free }}+4 s^{2}\left(\frac{k}{2 \pi} p_{j} \dot{x}^{j}\right)^{2}}}{2 s},
$$

with $H_{\text {free }}=\frac{1}{2} p_{j} p^{j}+\frac{1}{2}\left(\frac{k}{2 \pi}\right)^{2} \hat{x}^{j} \dot{x}_{j}$. Equation (8) reduces to a free Hamiltonian at $s=0$, i.e., at $a=1 / 2$. In view of Eqs. (5)-(8), we conclude that the $\mathrm{T}^{4}$ modes can be equivalently represented as a free system with statedependent world-sheet length $R=J+\mathbf{H} / 2$ (for $a=1 / 2$ ) or as an interacting one with fixed length $R=J$ (for $a=0$ ).

\section{RELATION TO $T \bar{T}$ DEFORMATIONS}

The form of Eq. (8) is that of a $T \bar{T}$ deformation of free bosons [40-42]. To understand why, let us review and in fact slightly generalize the construction of such deformations. Given two conserved local currents $j_{I}^{\alpha}, I=1,2$ the limit

$$
j_{1} j_{2}(x)=\lim _{y \rightarrow x} j_{1}^{\alpha}(x) j_{2}^{\beta}(y) \epsilon_{\alpha \beta},
$$

is well defined owing to the arguments of Ref. [40], and

$$
\left\langle j_{1} j_{2}\right\rangle=\left\langle j_{1}^{\alpha}\right\rangle\left\langle j_{2}^{\beta}\right\rangle \epsilon_{\alpha \beta} .
$$

Notice that we do not require any of the currents $j_{I}^{\alpha}$ to be chiral. A $T \bar{T}$ deformation corresponds to the case $j_{I}^{\alpha}=T^{\alpha I}$. Coupling $T^{\alpha I}$ to a $\mathrm{u}(1)$ current yields deformations of the type considered in Ref. [50]; " $J \bar{J}$ " deformations fall in this class too, by taking a current and its (conserved) Hodge dual. For such special choices of $j_{I}^{\alpha}$ the deformation has a simple effect on the spectrum [40,50], and, in particular, for a $T \bar{T}$ deformation of parameter $\alpha$, we have

$$
\partial_{\alpha} H_{n}=-H_{n} \partial_{R} H_{n},
$$

for a state $|n\rangle$ of energy $H_{n}$ and zero momentum [40]. From this it follows that $H_{n}(R, \alpha)=H_{n}\left(R-\alpha H_{n}, 0\right)$ : the deformation amounts to a state-dependent shift of the length, which can be described as a CDD factor $[41,42]$. This is also the effect induced on $p_{+}$by $a$-gauge transformations, cf. Eq. (7), which explains the form of Eq. (8). Gauge transformations and $T \bar{T}$ deformations should not be confused however: the former leave the spectrum invariant, while the latter correspond to changing $p_{+}$while leaving $R$ fixed or vice versa. Indeed the differential equation for $a$-gauge transformations is Eq. (7) rather than Eq. (11). Hence, our observation that the "flat" subsector of classical $\mathrm{AdS}_{3} \times \mathrm{S}^{3} \times \mathrm{T}^{4}$ strings is simply related to a free theory is 
unsurprising in view of Refs. [41,42]. It is remarkable, as we will see, that this extends to the full quantum level, not only for $\mathrm{T}^{4}$ modes but for the whole superstring background.

\section{WZW SPECTRUM}

The spectrum of the light-cone Hamiltonian can be constructed from the left and right Kač-Moody currents $[10,51-55]$, as we very briefly review here. In the left sector, we have $\mathrm{sl}(2)_{k+2}$ currents $L_{-n}^{0, \pm}, \operatorname{su}(2)_{k-2}$ currents $J_{-n}^{3, \mp}$, torus modes $\alpha_{-n}^{r}$ as well as their fermionic superpartners. They act on a vacuum $\left|\ell_{0}, j_{0}\right\rangle$ given by a lowest (highest) weight state of sl(2), respectively, su(2). A generic state is obtained by acting with positive energy modes of the currents $(n \geq 0)$ on the vacuum, e.g.,

$$
\prod_{i=1}^{\ell^{+}} L_{-n_{i}}^{+} \prod_{i=1}^{\ell^{-}} L_{-n_{i}}^{-} \prod_{i=1}^{j^{+}} J_{-n_{i}}^{+} \prod_{i=1}^{j^{-}} J_{-n_{i}}^{-} \prod_{i=1}^{M_{T}} \alpha_{-n_{i}}^{r_{i}}\left|\ell_{0}, j_{0}\right\rangle .
$$

Such a state has (left) energy $\ell=\ell_{0}+\delta \ell$ and (left) angular momentum $j=j_{0}-\delta j$ with $\delta \ell=\ell^{+}-\ell^{-}$and $\delta j=j^{-}-j^{+}$. Fermions can be added in a similar way and the usual subtleties arise depending on their boundary conditions; see e.g., Refs. [55,56] for details. Physical states are subject to restrictions, the most important being the mass-shell condition

$$
-\frac{\ell_{0}\left(\ell_{0}-1\right)}{k}+\frac{j_{0}\left(j_{0}+1\right)}{k}+N_{\mathrm{eff}}=0,
$$

where $N_{\text {eff }}=\sum_{j} n_{j}$ is the total mode number (which in the NS sector is shifted by $-1 / 2$ ). More general sectors of the spectrum can be described by spectral flow [10], though we will not consider them in this article. Similar expressions hold in the right sector, which we label with tildes. Imposing level-matching $N_{\text {eff }}=\tilde{N}_{\text {eff }}$ and $j_{0}=\tilde{\jmath}_{0}$, we finally get

$$
E-J=\sqrt{\left(2 j_{0}+1\right)^{2}+4 k N_{\text {eff }}}-\left(2 j_{0}+1\right)+\delta,
$$

where $\delta=\delta \ell+\delta \tilde{\ell}+\delta j+\delta \tilde{\jmath}+2$ and we solved Eq. (13). Notice that for BPS states $E=J$ and $N_{\text {eff }}=\delta=0$ [57].

\section{FREE-THEORY INTERPRETATION}

The spectrum of excitations over the BPS "vacuum" is simply related to a $T \bar{T}$-like deformation of a free theory. Consider a theory of eight free bosons with dispersion

$$
H(p)=\left|\frac{k}{2 \pi} p+\mu\right|, \quad \mu \in\{0,0,+1,-1\}^{\oplus 2} .
$$

This coincides [58] with the plane-wave dispersion of our string background $[6,56,59]$ which for the NS-NS background is exact [31,36]. Supersymmetry can be realized by adding eight fermions with the same masses $\mu$. Imposing boundary conditions on a circle of size $R_{\text {eff }}$, we have

$$
\mathbf{H}=\sum_{i} H\left(\frac{2 \pi n_{i}}{R_{\mathrm{eff}}}\right)=\frac{k(N+\tilde{N})}{R_{\mathrm{eff}}}+\sum_{i} \mu_{i} \operatorname{sgn}\left(n_{i}\right),
$$

where we split left- and right-movers. Notice that to remove the absolute value we have assumed that $R_{\text {eff }} \leq k$; we will see shortly why this is the case. If we now postulate the state-dependent length

$$
R_{\mathrm{eff}}=R_{0}+\frac{\mathbf{H}-\mathbf{m}}{2}, \quad \mathbf{m}=\sum_{i} \mu_{i} \operatorname{sgn}\left(n_{i}\right),
$$

and solve Eq. (16), we precisely reproduce the WZW lightcone energy (14) with the following identifications. First, $\mathbf{H}=E-J$ as in Eq. (6). Next $R_{0}=2 j_{0}+1$ is the $J$-charge of the BPS state in the R-R sector corresponding to the middle of the $\mathrm{T}^{4}$ Hodge diamond. Notice that taking $R_{0}$ to be the charge of a reference vacuum rather than the $J$-charge of the state itself mimics the dual spin-chain construction for $\mathrm{AdS}_{5} \times \mathrm{S}^{5}[35,60]$. Finally $\mathbf{m}=\delta$. Let us justify this. Notice that when no excitations on $\operatorname{AdS}_{3} \times \mathrm{S}^{3}$ are present, $\mu=0$ and Eq. (17) precisely describes a $T \bar{T}$ deformation [40-42]. Consider now a state with some $\mathrm{T}^{4}$ excitations over the BPS vacuum and a single $S^{3}$ mode, say $J_{-n}^{ \pm}$. For the charges to match, this should correspond to a boson with $p=2 \pi n / R_{\text {eff }} \geq 0$ and $\mu=\mp 1$; conversely, $\tilde{J}_{-\tilde{n}}^{ \pm}$ gives a boson with $p=-2 \pi \tilde{n} / R_{\text {eff }} \leq 0$ and $\mu= \pm 1$ [61]. This matches the identification of $\mu$ with the su(2)-spin of $\mathrm{S}^{3}$ excitations in the plane-wave limit [31]. The other bosons as well as the fermions can be similarly described and will be presented elsewhere [47]. Finally, notice that $R_{\text {eff }}=\ell_{0}+j_{0}$ with our identifications. The condition $R_{\text {eff }} \leq k$ which we used to remove the absolute values in Eq. (16) follows from the unitarity bounds of the WZW model [10]. Sectors with larger values of $R_{\text {eff }}$ should arise from spectral flow, see also Ref. [56] for a discussion of this fact in the plane-wave limit.

\section{S MATRIX AND BETHE-YANG EQUATIONS}

An energy-dependent shift of the length can be described as a CDD factor [43] to the S matrix, see Ref. [41]. This is also the case for the shift of Eq. (17) which corresponds to a CDD factor whose phase is

$$
\Phi_{j k}=p_{j} E_{k}-p_{k} E_{j}-p_{j} m_{k}+p_{k} m_{j}
$$

where $m_{j}=\mu_{j} \operatorname{sgn}\left(k p_{j}+2 \pi \mu_{j}\right)$. Starting from a free theory, we get a diagonal $\mathrm{S}$ matrix with elements $S_{j k}=\exp \left(\frac{i}{2} \Phi_{j k}\right)$. The Bethe-Yang equations follow immediately, 


$$
1=\exp \left(i p_{k} R_{0}\right) \prod_{j \neq k} S_{k j}=\exp \left(i p_{k} R_{\mathrm{eff}}\right),
$$

where in the last equation we used the level-matching condition $\sum p_{j}=0$. Given that $H$ and $m$ distinguish between left- and right-movers, it is convenient to treat such modes separately. We introduce labels " \pm " for particles having $\partial H / \partial p= \pm k / 2 \pi$, yielding four cases for the $\mathrm{S}$ matrix. We get

$S_{j k}^{++}=S_{j k}^{--}=1, \quad S_{j k}^{-+}=\exp \left(i \frac{k}{2 \pi} p_{j} p_{k}\right)=\frac{1}{S_{k j}^{+-}}$.

This illustrates the role of $\mathbf{m}$ : it makes the left-left and rightright scattering trivial, as we would expect in a theory where particles move at the speed of light. Notice that such scattering is much simpler than the one arising in Refs. [62,63], where nondiagonal and nonperturbative left-left and right-right $\mathrm{S}$ matrices appear. These expressions match the perturbative tree-level result for $S_{i j}^{ \pm \mp}$ of Ref. [44]. To compare our expressions, we should firstly take the results of Ref. [44] in the $a=0$ gauge; in that case, the length in the Bethe-Yang equations is the $J$-charge of the state-in contrast to our conventions, in which it is the $J$-charge of the BPS vacuum. Accounting for these different conventions is akin to going from the string-frame to the spin-chain frame $[18,28,29]$. With these identifications, the left-right and right-left $\mathrm{S}$ matrices match with Refs. [44,64]. Based on the integrability treatment of strings in flat space [65] it may appear surprising that our analysis relies solely on the Bethe-Yang equations (19) and does not require the mirror thermodynamic Bethe ansatz to account for finitesize effect, cf. Refs. $[19,66]$; this is all the more concerning given that this background features gapless excitations that usually lead to severe wrapping effects [67]. This simplification is due to supersymmetry: as the scattering is diagonal, wrapping corrections [68-70] to a state with momenta $p_{1}, \ldots p_{M}$ take a simple form

$$
\int \mathrm{d} \rho e^{-\varepsilon(\rho) L} \sum_{X}(-1)^{F_{X}} \prod_{j=1}^{M} S_{X j}\left(\rho, p_{j}\right),
$$

where $X$ is any virtual particle. Regardless of the details, here bosons and fermions come in pairs with identical dispersion and scattering, so that the integrand vanishes; this is the same argument that guarantees that BPS states are immune from wrapping corrections in Ref. [45].

\section{TOWARDS A DEFORMATION OF THE FULL ACTION}

A formula for the action of $T \bar{T}$ deformations of scalar field theories is known [42,71,72]. We briefly discuss two subtleties arising when applying such an approach here: firstly, our transformation involves the current $\mathbf{m}$; secondly, our free action has the $\mu$-dependent dispersion (15). Naïvely we would use Eq. (9) with one of the currents given by $j^{\alpha}$ such that $\int \mathrm{d} \sigma j^{0}=\mathbf{m}$; unfortunately, while such a conserved current exists in a free theory, it is nonlocal and Zamolodchikov's arguments [40] do not apply [73]. Alternatively we can ask whether the gaugefixed WZW action is the $T \bar{T}$ deformation of some simpler theory; this is also quite subtle. In the presence of several so(2) symmetries such as the ones rotating $z_{1,2}$ and $y_{3,4}$ the stress-energy tensor is not uniquely defined. To be concrete, we truncate our theory to the $S^{3}$ modes and introduce complex coordinates $y, \bar{y}$. The dispersion (15) can be reproduced by coupling $y, \bar{y}$ to a constant $\mathrm{u}(1)$ background gauge field $A^{\alpha}$. The Noether stress-energy tensor $T_{\mathrm{N}}^{\alpha \beta}$ is not gauge-invariant; adding improvement terms yields the Hilbert stress-energy tensor $T_{\mathrm{H}}^{\alpha \beta}$. The difference of the two $T \bar{T}$ operators is also of the form (9),

$$
T_{\mathrm{H}} \bar{T}_{\mathrm{H}}-T_{\mathrm{N}} \bar{T}_{\mathrm{N}}=\epsilon_{\alpha \beta} \epsilon_{I J} j^{\alpha ; I} T^{\beta J},
$$

where the two currents $j^{\alpha ; I}$ are related to the components of the constant gauge field

$$
j^{\alpha ; I}=i A^{I}\left(p^{\alpha} \bar{y}-\bar{p}^{\alpha} y\right) .
$$

Hence, we have at least two inequivalent $T \bar{T}$ deformations. A priori it is unclear which one is more natural; interestingly, a Hilbert-T $\bar{T}$ deformation relates the gauge-fixed GS action to a simple sigma model action for the sphere fields[74],

$$
\left.\mathbf{S}\right|_{\mathrm{S}^{3}}=\frac{k}{2 \pi} \int_{-\infty}^{+\infty} \mathrm{d} \tau \int_{0}^{R} \mathrm{~d} \sigma \eta^{\alpha \beta} \frac{D_{\alpha} y D_{\beta} \bar{y}}{1-y \bar{y}},
$$

with background gauge field $A^{\alpha}=g^{-1} \partial^{\alpha} g, g=\exp [i \sigma]$. The integrability of the classical $\mathrm{AdS}_{3} \times \mathrm{S}^{3} \times \mathrm{T}^{4}$ action suggests that (24) is classically integrable too.

\section{CONCLUSIONS AND OUTLOOK}

We have found evidence that superstrings on $\mathrm{AdS}_{3} \times$ $\mathrm{S}^{3} \times \mathrm{T}^{4}$ with NS-NS three-form flux are described by a simple integrable theory of eight relativistic bosons and fermions with dispersion (15) and S matrix (20) given by a CDD factor. For such a theory wrapping corrections cancel and the Bethe-Yang equations are exact, rather than asymptotic. While this description is strongly reminiscent of a $T \bar{T}$ deformation, constructing the appropriate perturbing operator is quite subtle. A number of questions immediately arise. Our construction here was limited to "unflowed" sector of the WZW model, corresponding to $R_{\text {eff }} \leq k$ in Eq. (16). It would be interesting to extend this to the $w$-th spectrally flowed sector corresponding to $w k<R_{\text {eff }} \leq(w+1) k$, see also Ref. [56] for a discussion of this in the plane-wave limit; notice that when the 
inequality is saturated the mass-gap in Eq. (16) vanishes and new gapless modes appear. We also restricted to states with vanishing total momentum (i.e., $N=\tilde{N}$ ). In light-cone gauge, winding sectors should also be included [11,37], which would modify our analysis and in particular Eq. (19). Finally, it is intriguing that the gauge-fixed WZW action is related to Eq. (24) and it would be worth exploring more such a sigma model. We will return to these questions in an upcoming publication [47]. It would also be worth extending this analysis to $\mathrm{AdS}_{3} \times \mathrm{S}^{3} \times \mathrm{S}^{3} \times \mathrm{S}^{1}$ backgrounds, whose integrability $[27,32,45]$ and WZW $[10,75]$ descriptions are well-established, as well as more general supersymmetric theories with diagonal scattering, where wrapping corrections are also expected to cancel. It looks less likely that this scenario might hold for mixed R-R and NS-NS backgrounds, as the S matrix is nontrivial in that case $[31,44]$, though one might hope that the first correction in the R-R flux is captured by Eqs. (16) and (17) with the exact mixed-flux dispersion [31,36] instead of Eq. (15). Such mixed-flux dynamics is particularly interesting as it captures a large part of the moduli space [76]. Describing strings on NS-NS backgrounds as simple integrable theories would have a number of interesting applications. As our description depends parametrically on the WZW level $k$ we could apply it to, e.g., the semiclassical limit $k \gg 1$ as well as to special cases such as the $k=1$ theory which was recently related to a symmetric-product orbifold CFT $[77,78]$. Interestingly, our dispersion (15) at $k=1$ precisely describes the single-excitation spectrum of the symmetricproduct orbifold CFT of $\mathrm{T}^{4}$ [79]. This might help us find an integrability description for symmetric-product orbifold
CFTs, cf. also Ref. [80]. It would also be interesting to extend this map beyond the spectrum: recently integrability techniques have been developed to compute three- [21] and higher-point $[22,23]$ functions, and even nonplanar corrections [24,25]. In $\mathrm{AdS}_{5} \times \mathrm{S}^{5}$ Lüscher-like wrapping effects make such computations very hard, while we have seen in Eq. (21) that those cancel here, at least for two-point functions. This, together with the wealth of data available might make NS-NS background an ideal playground for the hexagon bootstrap program [21-25].

\section{ACKNOWLEDGMENTS}

We are grateful to B. Hoare, O. Ohlsson Sax, B. Stefański, G. Tartaglino-Mazzucchelli, R. Tateo, and A. Tseytlin for comments on the manuscript. We thank A. Dei, K. Ferreira, B. Hoare, S. Komatsu, B. Oblak, G. TartaglinoMazzucchelli, L. Wulff, and K. Zarembo for useful related discussions. A. S. is grateful to R. Borsato, O. Ohlsson Sax, B. Stefański, and A. Torrielli, as well as to A. Dei and M. Gaberdiel for pleasant and fruitful collaboration on related topics. The work of M. B. is supported by the European Union's Horizon 2020 research and innovation program under the Marie Skłodowska-Curie Grant Agreement No. 665501 with the Research Foundation Flanders (FWO). M. B. is an FWO [PEGASUS $]^{2}$ Marie Skłodowska-Curie Fellow. A. S. acknowledges support by the ETH Career Seed Grant No. 0-20313-17, as well as partial support from the National Centre of Competence in Research "NCCR SwissMAP: The Mathematics of Physics", No. 141869, funded by the Swiss National Science Foundation.
[1] J. M. Maldacena, Adv. Theor. Math. Phys. 2, 231 (1998).

[2] E. Witten, Adv. Theor. Math. Phys. 2, 253 (1998).

[3] S. S. Gubser, I. R. Klebanov, and A. M. Polyakov, Phys. Lett. B 428, 105 (1998).

[4] M. Blau, J. M. Figueroa-O'Farrill, C. Hull, and G. Papadopoulos, J. High Energy Phys. 01 (2002) 047.

[5] M. Blau, J. M. Figueroa-O'Farrill, C. Hull, and G. Papadopoulos, Classical Quantum Gravity 19, L87 (2002).

[6] D. E. Berenstein, J. M. Maldacena, and H. S. Nastase, J. High Energy Phys. 04 (2002) 013.

[7] E. Witten, Commun. Math. Phys. 92, 455 (1984).

[8] V. G. Knizhnik and A. B. Zamolodchikov, Nucl. Phys. B247, 83 (1984).

[9] D. Gepner and E. Witten, Nucl. Phys. B278, 493 (1986).

[10] J. M. Maldacena and H. Ooguri, J. Math. Phys. (N.Y.) 42, 2929 (2001).

[11] G. Arutyunov and S. Frolov, J. Phys. A 42, 254003 (2009).

[12] N. Beisert et al., Lett. Math. Phys. 99, 3 (2012).

[13] A. Sfondrini, J. Phys. A48, 023001 (2015).
[14] A. B. Zamolodchikov and A. B. Zamolodchikov, Ann. Phys. (N.Y.) 120, 253 (1979).

[15] I. Bena, J. Polchinski, and R. Roiban, Phys. Rev. D 69, 046002 (2004).

[16] N. Beisert, Adv. Theor. Math. Phys. 12, 948 (2008).

[17] N. Beisert, B. Eden, and M. Staudacher, J. Stat. Mech. 2007, P01021.

[18] G. Arutyunov, S. Frolov, and M. Zamaklar, J. High Energy Phys. 04 (2007) 002.

[19] J. Ambjørn, R. A. Janik, and C. Kristjansen, Nucl. Phys. B736, 288 (2006).

[20] N. Gromov, V. Kazakov, S. Leurent, and D. Volin, Phys. Rev. Lett. 112, 011602 (2014).

[21] B. Basso, S. Komatsu, and P. Vieira, arXiv:1505.06745.

[22] B. Eden and A. Sfondrini, J. High Energy Phys. 10 (2017) 098.

[23] T. Fleury and S. Komatsu, J. High Energy Phys. 01 (2017) 130.

[24] B. Eden, Y. Jiang, D. le Plat, and A. Sfondrini, J. High Energy Phys. 02 (2018) 170. 
[25] T. Bargheer, J. Caetano, T. Fleury, S. Komatsu, and P. Vieira, arXiv:1711.05326.

[26] A. Babichenko, B. Stefański, Jr., and K. Zarembo, J. High Energy Phys. 03 (2010) 058.

[27] A. Cagnazzo and K. Zarembo, J. High Energy Phys. 11 (2012) 133.

[28] R. Borsato, O. O. Sax, and A. Sfondrini, J. High Energy Phys. 04 (2013) 113.

[29] R. Borsato, O. O. Sax, A. Sfondrini, B. Stefański, Jr., and A. Torrielli, J. High Energy Phys. 08 (2013) 043.

[30] R. Borsato, O. O. Sax, A. Sfondrini, and B. Stefański, Jr., Phys. Rev. Lett. 113, 131601 (2014).

[31] T. Lloyd, O. O. Sax, A. Sfondrini, and B. Stefański, Jr., Nucl. Phys. B891, 570 (2015).

[32] R. Borsato, O. O. Sax, A. Sfondrini, and B. Stefański, Jr., J. Phys. A 48, 415401 (2015).

[33] R. Borsato, O. O. Sax, A. Sfondrini, B. Stefański, Jr., and A. Torrielli, Phys. Rev. D 88, 066004 (2013).

[34] R. Borsato, O. O. Sax, A. Sfondrini, B. Stefański, Jr., and A. Torrielli, J. Phys. A 50, 024004 (2017).

[35] J. A. Minahan and K. Zarembo, J. High Energy Phys. 03 (2003) 013.

[36] B. Hoare, A. Stepanchuk, and A. Tseytlin, Nucl. Phys. B879, 318 (2014).

[37] G. Arutyunov and S. Frolov, J. High Energy Phys. 02 (2005) 059.

[38] G. Arutyunov and S. Frolov, J. High Energy Phys. 01 (2006) 055.

[39] G. Arutyunov, S. Frolov, and M. Zamaklar, Nucl. Phys. B778, 1 (2007).

[40] A. B. Zamolodchikov, arXiv:hep-th/0401146.

[41] F. A. Smirnov and A. B. Zamolodchikov, Nucl. Phys. B915, 363 (2017).

[42] A. Cavaglià, S. Negro, I. M. Szécsényi, and R. Tateo, J. High Energy Phys. 10 (2016) 112.

[43] L. Castillejo, R. H. Dalitz, and F. J. Dyson, Phys. Rev. 101, 453 (1956).

[44] B. Hoare and A. A. Tseytlin, Nucl. Phys. B873, 682 (2013).

[45] M. Baggio, O. O. Sax, A. Sfondrini, B. Stefaski, and A. Torrielli, J. High Energy Phys. 04 (2017) 091.

[46] Such a CDD factor first appeared in Ref. [39] in the context of uniform light-cone gauge transformations.

[47] A. Dei and A. Sfondrini, arXiv:1806.00422.

[48] M. Cvetič, H. Lü, C. N. Pope, and K. S. Stelle, Nucl. Phys. B573, 149 (2000).

[49] L. Wulff, J. High Energy Phys. 07 (2013) 123.

[50] M. Guica, arXiv:1710.08415.

[51] A. Giveon, D. Kutasov, and N. Seiberg, Adv. Theor. Math. Phys. 2, 733 (1998).

[52] A. Pakman, J. High Energy Phys. 01 (2003) 077.

[53] D. Israel, C. Kounnas, and M. P. Petropoulos, J. High Energy Phys. 10 (2003) 028.

[54] S. Raju, Phys. Rev. D 77, 046012 (2008).
[55] K. Ferreira, M. R. Gaberdiel, and J. I. Jottar, J. High Energy Phys. 07 (2017) 131.

[56] A. Dei, M. R. Gaberdiel, and A. Sfondrini, arXiv:1805 .09154 .

[57] This comes about slightly differently in the R and NS sectors and requires care with the GSO projection.

[58] The four modes with $\mu=0$ correspond to $\mathrm{T}^{4}$ directions; introducing complex coordinates $z, \bar{z}, y, \bar{y}$ for the $\mathrm{AdS}_{3} \times S^{3}$ transverse fields, two fields $(z, y)$ have $\mu=1$ and their conjugate have $\mu=-1$.

[59] J. G. Russo and A. A. Tseytlin, J. High Energy Phys. 04 (2002) 021.

[60] N. Beisert and M. Staudacher, Nucl. Phys. B727, 1 (2005).

[61] Zero-modes require some care. In the WZW model $J_{0}^{+}, \tilde{J}_{0}^{+}$ annihilate the vacuum; correspondingly, for $\mu=1(\mu=-1)$ only the left-moving (right-moving) zero mode survive.

[62] A. B. Zamolodchikov and A. B. Zamolodchikov, Nucl. Phys. B379, 602 (1992).

[63] P. Fendley, H. Saleur, and A. B. Zamolodchikov, Int. J. Mod. Phys. A 08, 5751 (1993).

[64] Ibid. $S_{i j}^{ \pm \pm}$is seemingly nontrivial, at least for mixed-flux backgrounds; however, carefully taking the pure-NS-NS limit as explained around Eq. (3.24) there, one finds $S_{i j}^{ \pm \pm}=1$, coherently with the expectation from perturbation theory.

[65] S. Dubovsky, R. Flauger, and V. Gorbenko, J. High Energy Phys. 09 (2012) 133.

[66] G. Arutyunov and S. Frolov, J. High Energy Phys. 12 (2007) 024.

[67] M. C. Abbott and I. Aniceto, Phys. Rev. D 93, 106006 (2016).

[68] M. Lüscher, Commun. Math. Phys. 104, 177 (1986).

[69] M. Lüscher, Commun. Math. Phys. 105, 153 (1986).

[70] Z. Bajnok and R. A. Janik, Nucl. Phys. B807, 625 (2009).

[71] R. Tateo, in Proceedings of IGST2017 (2017), https://www .phys.ens.fr/ igst17/timetable.html.

[72] R. Conti, L. Iannella, S. Negro, and R. Tateo, .

[73] Additionally here extended supersymmetry results in a degenerate spectrum, which also prevents us from straightforwardly applying Zamolodchikov's arguments.

[74] Here the Hilbert- $T \bar{T}$ deformation leads to formulas similar to Cavaglià et al. with $\partial_{\alpha} \rightarrow D_{\alpha}$.

[75] L. Eberhardt, M. R. Gaberdiel, R. Gopakumar, and W. Li, J. High Energy Phys. 03 (2017) 124.

[76] O. O. Sax and B. Stefański, Jr. (to be published).

[77] G. Giribet, C. Hull, M. Kleban, M. Porrati, and E. Rabinovici, arXiv:1803.04420.

[78] M. R. Gaberdiel and R. Gopakumar, arXiv:1803.04423.

[79] A. Sfondrini, in Proceedings of IGST2017 (2017), https:// www.phys.ens.fr/ igst17/timetable.html.

[80] A. Pakman, L. Rastelli, and S. S. Razamat, J. High Energy Phys. 10 (2009) 034. 